\title{
A semiclassical approach to the ground state and density oscillations of quantum dots
}

\author{
A. Puente, M. Casas and Ll. Serra \\ Departament de Física, Universitat de les Illes Balears, E-07071 Palma de Mallorca, Spain
}

(19 August 1999)

\begin{abstract}
A semiclassical Thomas-Fermi method, including a Weizsäcker gradient term, is implemented to describe ground states of two dimensional nanostructures of arbitrary shape. Time dependent density oscillations are addressed in the same spirit using the corresponding semiclassical time-dependent equations. The validity of the approximations is tested, both for ground state and density oscillations, comparing with the available microscopic Kohn-Sham solutions.
\end{abstract}

PACS 73.20.Dx, 72.15.Rn 


\section{INTRODUCTION}

Two-dimensional quantum dots, and semiconductor nanostructures in general, are examples of artificial systems in which, ultimately, we may seek to produce and control electronic quantum properties. Sometimes they are referred to as artificial atoms and in fact, it has been proved that the electronic structure of circular quantum dots resembles in some aspects the shell structure found in atoms and nuclei. Particularly relevant in this sense are the Coulomb blockade measurements that provide direct acces to the electronic energy levels by adding electrons one by one to the quantum dot [1]. The magic numbers measured in vertical quantum dots [2], corresponding to maxima in the addition energies, have also shown clear evidences of shell structure. Absorption measurements in the far-infrared region [3, [4] as well as experiments using sophisticated light scattering techniques [5,6] have probed both charge density (CDE) and spin density (SDE) excitations and have shown that parabolic confinement is closely satisfied for small size dots.

Interest in the quantum dot community has recently focussed on the properties of deformed nanostructures. For instance, elliptic quantum dots have been investigated in Refs. [7 13. Particularly, in Refs. [11,12] some of us have addressed the collective oscillations of deformed dots where, in addition to density and spin modes, it has been predicted the existence of orbital current modes at low energies. This interest in deformed dots is motivated by the advances in nanofabrication techniques, that allow to produce quantum dots of many different shapes.

Microscopic theoretical approaches, like Hartree [14], Hartree-Fock [15] and density functional [16 18, let alone exact diagonalizations [19,20], get very demanding computationally for increasing number of electrons, especially in the symmetry unrestricted case. It is thus interesting to develop a semiclassical approach, dealing only with the total density, for which the computational effort is not much dependent on the number of electrons. This is our purpose in this paper. Semiclassical Thomas-Fermi models have been already used in the field, for instance in Refs. [21 24]. In particular, Ref. [23] provides a quite rigorous presentation of the theory. However, previous works deal only with the semiclassical ground state and mostly for circular symmetry. Here we emphasize the application to deformed structures and concentrate on the oscillation modes. We do not include in this paper magnetic field since this requires a non trivial generalization of the theory that we plan to develop in a separate contribution.

The structure of the paper is as follows. In Sec. II we discuss the ground state in our semiclassical approach and compare with available microscopic Kohn-Sham (KS) results. Section III introduces the formalism for the time-dependent oscillations. Results for the oscillation modes are discussed in Sec. IV. Finally the conclusions are drawn in Sec. V.

\section{GROUND STATES}

\section{A. Definition of energy functional}

Using Density Functional Theory in a local approximation we write the total energy in terms of the electronic density $\rho(\mathbf{r})$ as $E[\rho]=\int d \mathbf{r} \mathcal{E}[\rho]$, with the energy density separating as 


$$
\mathcal{E}[\rho]=\tau[\rho]+\frac{1}{2} v_{H}(\mathbf{r}) \rho+\mathcal{E}_{X C}(\rho)+v_{\text {ext }}(\mathbf{r}) \rho
$$

The different terms are the kinetic energy density $\tau[\rho]$, the Hartree potential $v_{H}(\mathbf{r})=$ $\int d \mathbf{r}^{\prime} \frac{\rho\left(\mathbf{r}^{\prime}\right)}{\left|\mathbf{r}-\mathbf{r}^{\prime}\right|}$, the exchange-correlation energy density $\mathcal{E}_{X C}(\rho)$ and the external confining potential $v_{\text {ext }}(\mathbf{r})$. The Thomas-Fermi approximation in two dimensions (2D) yields the following kinetic energy density 25]

$$
\tau_{T F}[\rho]=\frac{\hbar^{2}}{2 m}\left(\pi \rho^{2}+\frac{5}{12} \nabla^{2} \rho\right)
$$

By analogy with the Weizäcker term for the 3D kinetic energy functional, we have added a gradient term that gives the exact kinetic energy for a single electron

$$
\tau_{W}[\rho]=\frac{\hbar^{2}}{2 m} \lambda \frac{(\nabla \rho)^{2}}{\rho},
$$

with $\lambda=1 / 4$. Our total Thomas-Fermi-Weizsäcker (TFW) kinetic functional is then $\tau=$ $\tau_{T F}+\tau_{W}$. It is worth to point out that the first non-vanishing gradient correction in $2 \mathrm{D}$ is not known from a rigorous semiclassical expansion and therefore we have introduced empirically the Weizsäcker-like term. We will show later that the results are not sensitive to the precise value of the coefficient $\lambda$. For the sake of comparison, we recall here that the KS method provides the exact kinetic energy by means of a set of single particle orbitals $\left\{\varphi_{i}\right\}$ as

$$
\tau(\mathbf{r})=\frac{\hbar^{2}}{2 m} \sum_{i, o c c .}\left|\nabla \varphi_{i}(\mathbf{r})\right|^{2} .
$$

For the exchange-correlation energy $\mathcal{E}_{X C}$ we have used the same functional of Ref. [17], based on the Tanatar and Ceperley calculation for the uniform gas [26]. Specifically, in modified atomic units [27], it is written as

$$
\mathcal{E}_{X C}(\rho)=-\frac{4}{3} \sqrt{\frac{2}{\pi}} \rho^{3 / 2}+\frac{1}{2} a_{0} \rho \frac{1+a_{1} x}{1+a_{1} x+a_{2} x^{2}+a_{3} x^{3}},
$$

where $x=(\pi \rho)^{-1 / 4}$ and the $a_{i}$ coefficients are given in Ref. 26]. Notice that in this formalism we always assume perfect spin degeneracy in the ground state in order to obtain a single chemical potential for both spin components (see below).

The ground state density is determined from the minimization (Euler-Lagrange) equation, with the contraint of particle number conservation

$$
\frac{\delta}{\delta \rho}\left(E[\rho]-\mu \int d \mathbf{r} \rho\right)=0
$$

Specifically, this reads

$$
\begin{aligned}
-2 \lambda \frac{\hbar^{2}}{2 m} \nabla^{2} \rho & +\lambda \frac{\hbar^{2}}{2 m} \frac{(\nabla \rho)^{2}}{\rho} \\
& +\left(v_{\text {ext }}+v_{H}+\frac{\partial \mathcal{E}_{X C}}{\partial \rho}+2 \pi \frac{\hbar^{2}}{2 m} \rho\right) \rho=\mu \rho .
\end{aligned}
$$


By making the transformation $\psi=\sqrt{\rho}$ this equation may be written in the familiar form of a Schrödinger like equation

$$
-4 \lambda \frac{\hbar^{2}}{2 m} \nabla^{2} \psi+V \psi=\mu \psi
$$

where we have defined the semiclassical effective potential $V=v_{e x t}+v_{H}+\frac{\partial \mathcal{E}_{X C}}{\partial \rho}+2 \pi \frac{\hbar^{2}}{2 m} \rho$. The chemical potential $\mu$ plays the role of the eigenvalue in Eq. (8). Written in this way, we may now use the algorithms developed to solve the KS equation in arbitrary 2D confinements [11]. Our method is based on a discretization of the $x y$ plane in a grid of uniformly spaced points. Then, the total number of grid points, not the number of electronic orbitals as in KS theory, determines the computational cost of the problem. Typically, we use grids ranging from $50^{2}$ to $100^{2}$ points.

\section{B. Results and comparison with Kohn-Sham}

In this subsection we show results for the semiclassical ground states in different confining potentials, focussing especially on the comparison with the corresponding microscopic $\mathrm{KS}$ results in order to prove the validity of the approximations. Since the microscopic calculation for medium and large sizes is computationally feasible only when circular symmetry is imposed to the system, we will begin by considering the cases of circular parabolas and disks of jellium. In these cases we compare with the KS radial solution [17. Then we analyze one case with deformation, namely the deformed parabola with $N=20$, and compare with a symmetry unrestricted microscopic calculation [11].

\section{Circular parabolas}

The confining potential in this case is (we will use modified atomic units [27] for the rest of the paper)

$$
v_{\text {ext }}(r)=A_{0}+\frac{1}{2} \omega_{0}^{2} r^{2}
$$

The constants $A_{0}$ and $\omega_{0}$ are usually parameterized to reproduce the potential and its curvature at the origin of a jellium disk (see below) with $N_{p}$ positive charges as $A_{0}=$ $-2 \sqrt{N_{p}} / r_{s}$ and $\omega_{0}^{2}=1 /\left(\sqrt{N_{p}} r_{s}^{3}\right)$, with $r_{s}$ the radius per unit charge. Figure 1 shows the densities obtained taking $r_{s}=1.51 a_{0}^{*}$. Panels (a) and (b) prove that the semiclassical density closely adjusts to the microscopic one for varying electron number in a fixed parabola and for a varying parabola curvature at fixed electron number, respectively. As is well known from atomic physics, the TFW density averages the microscopic shell oscillations in the inner region. Panel (c) shows that the coefficient $\lambda$ controls the surface width of the semiclassical density. The value $\lambda=1 / 4$ provides a reasonable approximation to the KS density tails in all cases. However, it is clear that if this coefficient is allowed to vary a better fit of the densities may be obtained. We have not followed this procedure since the fitted value of $\lambda$ would be different for each type of confining potential (see next subsections). 
In Fig. 2 we compare total energies per electron in the two approaches. The fit of energies is amazingly good; the deviation of the TFW energies with respect to KS being less than $0.5 \%$ for all cases shown in Fig. 2. Although the high precission of the TFW model for this quantity may seem a bit surprising, we recall that the method looks for the variational minimum of total energy. Therefore, this is a priori the best value of the model. We have made the comparison for magic electron numbers, corresponding to closed shell configurations. This is the reason why shell oscillations with size are not visible in the microscopic results of Fig. 2(a). The total energies are almost independent of $\lambda$ for reasonable values of this coefficient. The results for $N=42$ and $\lambda=0.25,0.5$ and 1.5 are $E / N=-4.033,-4.025$ and $-4.014 \mathrm{H}^{*}$, respectively.

\section{Jellium disks}

Another external potential with circular symmetry is that of a uniformly charged disk. Defining the positive charge density (jellium density) in terms of the $r_{s}$ parameter $\rho_{j}=$ $1 /\left(\pi r_{s}^{2}\right)$ and for a disk with radius $R$, the potential is

$$
v_{\text {ext }}(r)=\left\{\begin{array}{ll}
-\frac{4}{\pi} \frac{R}{r_{s}^{2}} E(r / R) & \text { if } \quad r \leq R \\
-\frac{4}{\pi} \frac{r}{r_{s}^{2}}\left[E(R / r)-\left(1-\left(\frac{R}{r}\right)^{2}\right) K(R / r)\right] & \text { if } \quad r \geq R
\end{array},\right.
$$

where $E$ and $K$ are the elliptic integrals. Assuming a uniformly charged disk the number of positive charges $N_{p}$ is related to the disk radius by $R=r_{s} \sqrt{N_{p}}$.

Contrary to the parabola, the jellium potential forces the electronic density to saturate inside the dot. This is obviously due to the charge screening effect, that energetically favors the cancellation of charges. Figure 3 shows the electronic densities of neutral disks with

$r_{s}=1.51 a_{0}^{*}$ as a function of size. The agreement between both models is rather good. As for the parabolas, the TFW densities average the oscillations of the KS ones but now they rapidly saturate inside the dot. Close to the edge, the TFW densities present an small oscillation. This is similar to the Friedel-type oscillations found in metal surfaces and is enhanced by potentials that abruptly vanish at the edge, as the jellium one. Also shown in Fig. 3 (panel b) are the energies per electron of the neutral disks, that are also very well reproduced by the semiclassical model.

From Fig. 3 we see that the TFW density tails slightly overestimate the KS ones, with the used value of $\lambda$. This implies that the number of electrons that are spilling out of the jellium disk will be slightly enhanced in the semiclassical method. This spill out mechanism is known from cluster physics to be of fundamental importance for a proper description of the optical properties [28]. The fact that our TFW method correctly includes spill out gives us some confidence in its use for the description of time dependent density oscillation (see Sec. IV).

\section{Deformed parabola for $N=20$}

The third case we have considered is an elliptical dot, confined by an anisotropic parabola, 


$$
v_{\text {ext }}(\mathbf{r})=\frac{1}{2} \omega_{0}^{2} \frac{4}{(1+\beta)^{2}}\left(x^{2}+\beta^{2} y^{2}\right) .
$$

The parameter $\beta$ gives the ratio of parabola coefficients in $y$ and $x$ directions, i.e., writing the external potential as $v_{\text {ext }}(\mathbf{r})=\frac{1}{2}\left(\omega_{x}^{2} x^{2}+\omega_{y}^{2} y^{2}\right)$, we have $\beta=\omega_{y} / \omega_{x}$. At the same time the centroid $\left(\omega_{x}+\omega_{y}\right) / 2$ is kept fixed at the value $\omega_{0}$, defined as for the circular parabola $\omega_{0}^{2}=1 /\left(r_{s}^{3} \sqrt{N_{p}}\right)$.

Since the KS problem is much more involved than for the preceding circular potentials, we restrict here the comparison to the $N=20$ electrons case, with $N_{p}=20$, and consider four deformations $\beta=0.875,0.75,0.625$ and 0.5 . Figure 4 shows the densities corresponding to these four deformations as well as the circularly symmetric case $(\beta=1)$ for completeness. It is seen that, quite nicely, the elliptic contour lines of the TFW density follow in average the equidensity regions of the KS result. For $\beta=1$ and $\beta=0.875$ the structure of equidensity regions of both models is very similar. For lower values of $\beta$ the microscopic model yields an incipient electron localization that the semiclassical model is obviously not able to reproduce; it gives nevertheless the correct average value. We conclude that the semiclassical model reproduces the average density distributions also in non circular systems. Total energies, given in Tab. I, are also in excellent agreement with the KS ones.

\section{TIME DEPENDENT EQUATIONS}

To derive the time dependent equations in the semiclassical approximation we will follow the fluid dynamical approach. This method has been used in nuclear physics [29] and, more recently, also for electronic oscillations in atomic clusters [30]. The variational derivation of these equations can be found in the book by Ring and Schuck [29]. Here we will just point out the essential ingredients and particular details for the application to our case.

The essential assumption is that all the single particle orbitals evolve in time with a common complex phase as

$$
\varphi_{i}(\mathbf{r}, t)=\varphi_{i}^{(0)}(\mathbf{r}, t) e^{i s(\mathbf{r}, t)}
$$

where both $\varphi_{i}^{(0)}(\mathbf{r}, t)$ and $s(\mathbf{r}, t)$ are assumed to be real functions. With (12) the kinetic energy separates in two contributions

$$
T=T_{\text {intr }}+\frac{1}{2} \int d \mathbf{r} \rho \mathbf{u}^{2}
$$

an intrinsic one $T_{\text {intr }}=\frac{1}{2} \sum_{i}\left|\nabla \varphi_{i}^{(0)}\right|^{2}$ and another associated to the common velocity field $\mathbf{u}=\nabla s$. Generalizing this separation we may write for the total energy (expectation value of the Hamiltonian $H$ )

$$
\mathcal{H} \equiv\langle H\rangle=E_{\text {intr }}+\frac{1}{2} \int d \mathbf{r} \rho \mathbf{u}^{2}
$$

In our density functional approach the intrinsic energy is in fact given by the energy functional $E_{\text {intr }}=E[\rho]$. Noticing now that $\rho$ and $s$ are conjugated canonical variables, Hamilton's equations are 29] $\dot{\rho}=\frac{\delta \mathcal{H}}{\delta s},-\dot{s}=\frac{\delta \mathcal{H}}{\delta \rho}$. Specifically, these read 


$$
\begin{aligned}
\dot{\rho} & =-\nabla(\rho \nabla s) \\
-\dot{s} & =\frac{1}{2}(\nabla s)^{2}+\frac{\delta E[\rho]}{\delta \rho} .
\end{aligned}
$$

The two Eqs. (15) yield the time evolution of the semiclassical variables $\rho$ and $s$, and thus they are our required input to model a semiclassical dynamics in quantum dots. As happens for the ground state equation, we may still transform Eqs. (15) to a form similar to the microscopic equation. In fact, defining the time dependent complex function $\psi=\sqrt{\rho} e^{i s}$ both Eqs. (15) reduce to

$$
i \frac{\partial \psi}{\partial t}=-\frac{1}{2} \Delta \psi+\left(\frac{\delta E[\rho]}{\delta \rho}+\frac{1}{2} \frac{\Delta \sqrt{\rho}}{\sqrt{\rho}}\right) \psi,
$$

i.e., an equation identical to the time-dependent $\mathrm{KS}$ equation if we identify the contribution within brackets in the right hand side with the potential term.

In the preceding discussion we have assumed, for simplicity, that both spin densities are oscillating in phase, and thus identically to the total density. The formalism, however, can also account for the situation in which both spin densities $\rho_{\eta}(\eta=\uparrow, \downarrow)$ oscillate out of phase, as happens for instance in spin modes. In this case we just need to assume the semiclassical approximation for each spin fluid, with the Coulombic coupling terms between them and generalize the exchange-correlation contribution to the locally polarized case using the exchange interpolation formula [26]. Defining $\psi_{\eta}=\sqrt{\rho}_{\eta} e^{i s_{\eta}}$ the two equations are

$$
i \frac{\partial \psi_{\eta}}{\partial t}=-\frac{1}{2} \Delta \psi_{\eta}+\left(\frac{\delta E\left[\rho_{\uparrow}, \rho_{\downarrow}\right]}{\delta \rho_{\eta}}+\frac{1}{2} \frac{\Delta \sqrt{\rho}_{\eta}}{\sqrt{\rho}_{\eta}}\right) \psi_{\eta} .
$$

In this paper we apply the time dependent equations to obtain the linear response frequencies corresponding to dipole charge density (CDE) and spin density (SDE) excitations of general 2D nanostructures. These are normal modes of oscillation and the technique to obtain them is simply to apply an small instant perturbation on the ground state and take this as initial condition for the time simulation. For the CDE the initial perturbation is simply a rigid translation of the total electronic density, given by operator $\mathcal{T}(\mathbf{a})$, with a the vector displacement. For the SDE we apply opposite translations for spin up and down densities, $\mathcal{T}_{\eta}\left(\mathbf{a}_{\eta}\right)$, with $\mathbf{a}_{\uparrow}=-\mathbf{a}_{\downarrow}$. After this, we keep track of the time evolution of the dipole moments $d_{\eta}(t)=\langle\mathbf{r}\rangle_{\eta} \cdot \hat{\mathbf{e}}$, where $\hat{\mathbf{e}}$ is a unitary vector in the direction of the displacement, and

$$
\langle\mathbf{r}\rangle_{\eta}=\frac{1}{N_{\eta}} \int d \mathbf{r} \rho_{\eta}(\mathbf{r}, t) \mathbf{r}
$$

with $N_{\uparrow}, N_{\downarrow}$ the number of electrons with spin up and down, respectively. A frequency analysis of the total dipole moment $d_{\uparrow}+d_{\downarrow}$ for the CDE, and of the spin dipole moment $d_{\uparrow}-d_{\downarrow}$ for the SDE, finally yields the oscillation frequencies [11].

\section{RESULTS OF DENSITY OSCILLATIONS}

We present in this section the spectra obtained within the semiclassical formalism. We consider density and spin dipole oscillations and, as in Sec. I, we emphasize the comparison 
with the corresponding KS results. One case is taken as representative for the three types of confining potentials for which we have already discussed the ground state.

\section{Circular parabola with $N=56$}

Figure 5 shows the CDE and SDE for the parabola with $N=56$ and $N_{p}=40$. The results have been plotted in a logarithmic arbitrary vertical scale (each tick marks an order of magnitude). First, we notice that in the CDE the semiclassical spectrum (and also the KS one) yield a single frequency that coincides with the parameter $\omega_{0}$ of the confining potential. We thus conclude that time-dependent TFW satisfies well the generalized Kohn's theorem [19]. This theorem states that the exact dipole CDE for parabolic confinement is a single peak at the parabola frequency $\omega_{0}$. The more intense SDE's lie at lower energy because the residual interaction is attractive in this channel [31]. The semiclassical model does not include the coupling to particle-hole excitations, that leads to an important fragmentation of the KS spectrum. Nevertheless, it reproduces some fragmentation of the collective strength, contained in two dominant peaks at $\approx 0.05 \mathrm{H}^{*}$ and $\approx 0.19 \mathrm{H}^{*}$, that nicely correspond to very intense KS excitations.

We remark that the microscopic results in Fig. 5 (and also those of Fig. 6 below) have been obtained by using the perturbative random-phase approximation (RPA) in a particlehole basis [18] while the TFW results were obtained, as explained in Sec. IV, from a frequency analysis of the real time signal. The RPA calculation provides a very high frequency resolution, even for low intensity peaks. On the contrary, the analysis of the time signal is not able in some cases to discriminate the low intensity peaks because of the limitations of a discrete time sampling and a finite total time window.

\section{Circular jellium with $N=58$}

This is shown in Fig. 6. In this case generalized Kohn's theorem does not hold since the external potential is not of parabolic type. As a consequence the dipole charge oscillation couples to the relative motion and the spectrum is generally fragmented, with peak energies depending on the number of electrons [31]. The disk potential behaves quadratically close to the disk center but it deviates for points closer to the edge. Fig. 6 shows the $\omega_{0}$ value for the quadratic behaviour at the origin. The semiclassical model reproduces the blue shift from $\omega_{0}$ although it slightly underestimates its quantitative value. This may be traced back to the slight difference in spill out mentioned in Sec. II.B.2. The TFW model yields a greater spill out and thus produces a softer oscillation mode. The absence of an exact Kohn mode manifests with an important fragmentation, partly reproduced in the TFW model. In the SDE of Fig. 6 the situation is similar to the circular parabola. The semiclassical spectrum reproduces the dominant collective peaks, and has less fragmentation than KS.

\section{Deformed parabola}

Figure 7 shows the results for an anisotropic parabola with $N=20, N_{p}=20$ and different deformations. The two upper panels correspond to the CDE for $\beta=0.5$ and 0.75 . They 
prove that also in deformed parabolas the generalized Kohn's theorem is well satisfied by time dependent TFW. In this case the parabolas in $x$ and $y$ directions are different and, in fact, the oscillation in these two directions is at frequency $\omega_{x}$ and $\omega_{y}$, respectively. For the two other deformations not shown in the figure $(\beta=0.625,0.875)$ generalized Kohn's theorem is equally satisfied. Therefore, as for TDLDA, TDTFW fulfills the exact property for dipole charge oscillations saying that the two center of mass coordinates $X=\sum_{i} x_{i}$ and $Y=\sum_{i} y_{i}$ oscillate with the frequencies $\omega_{x}$ and $\omega_{y}$, respectively [32].

The four lower panels of Fig. 7 show the SDE spectra for the four deformations considered. The $x-y$ splitting in the spin channel is nicely reproduced, as a function of deformation within the TFW model. Fragmentation is present in both models, although TFW is overestimating the collective strength at high energy for $\beta=0.75$ and $\beta=0.875$. We attribute this to the rather small electron number of this $\operatorname{dot}(N=20)$, for which TFW is surely less accurate than for large sizes. We remark again that the lower energy (and more intense) peak, as well as the $x-y$ splitting are correctly reproduced.

To finish this section and in order to emphasize the power of the semiclassical method we reproduce in Fig. 8 a case that is beyond the present capability of microscopic KS calculations. It corresponds to 72 electrons in a deformed parabola, with $N_{p}=72$ and $r_{s}=1.51 a_{0}^{*}$. Since the CDE corresponds only to $\omega_{x}$ and $\omega_{y}$, according to Kohn's theorem, we display the result of the SDE for $\beta=0.75$, as well as the circular one for comparison.

\section{CONCLUSIONS}

In this paper we have discussed a semiclassical approach to the ground state and density oscillations of 2D nanostructures. The method has been implemented for the general case in which no spatial symmetry is required. The validity of both ground state and dipole oscillation descriptions has been checked by systematically comparing the semiclassical TFW results with the corresponding KS ones. We have shown that the TFW densities closely follow the KS ones, averaging the shell oscillations, for different types of external confinings: circular and deformed parabolas and jellium disks for which the density saturates. Besides, the TFW model reproduces the KS energies with great accuracy. The dependence of the energy with the value of the Weizsäcker coefficient is very small. This coefficient controls the density tail and electronic spill out for jellium disks. We have shown that the value $\lambda=1 / 4$ provides a good overall fit.

Dipole charge density and spin density oscillations have been analyzed by using the time dependent semiclassical equations. In circular parabolic confinements we have shown that the semiclassical spectra satisfy well the generalized Kohn's theorem for charge density excitations, while in neutral jellium disks it yields a blue shift similar to the KS one. In elliptic dots the generalized Kohn's theorem is satisfied as well. For spin density excitations the TFW model is able to reproduce the dominant peaks of the spectrum and the splitting associated with deformation in elliptic dots.

In general, we have shown that the semiclassical Thomas-Fermi-Weizsäcker model provides a reliable tool to obtain accurate approximations to the ground state and linear oscillations of quantum dots. This opens the possibility to use it in order to explore a great 
variety of confining potentials with different geometries and for large sizes.

This work was performed under Grant No. PB95-0492 from CICYT, Spain. 


\section{REFERENCES}

[1] R. C. Ashoori, Nature (London) 379, 413 (1996).

[2] S. Tarucha, D. G. Austing, T. Honda, R. J. Van der Hage, L. P. Kouwenhoven, Phys. Rev. Lett. 77, 3613 (1996).

[3] Ch. Sikorski and U. Merkt, Phys. Rev. Lett. 62, 2164 (1989).

[4] T. Demel, D. Heitmann, P. Grambow, and K. Ploog, Phys. Rev. Lett. 64, 7881990.

[5] D. J. Lockwood, P. Hawrylak, P. D. Wang, C. M. Sotomayor-Torres, A. Pinkzuk, And B. S. Dennis, Phys. Rev. Lett. 77, 354 (1996).

[6] C. Schüller, K. Keller, G. Biese, E. Ulrichs, L. Rolf, D. Heitmann, and K. Eberl, Phys. Rev. Lett. 80, 2673 (1998).

[7] D. G. Austing, S. Sasaki, S. Tarucha, S. M. Reimann, M. Koskinen, M. Manninen, LANL preprint cond-mat/9905135.

[8] A. V. Madhav and T. Chakraborty, Phys. Rev. B 49, 8163 (1994).

[9] K. Hirose and N. S. Windgreen, Phys. Rev. B 59, 4604 (1999).

[10] C. Yannouleas and U. Landman, Phys. Rev. Lett. 82, 5325 (1999).

[11] A. Puente and Ll. Serra, Phys. Rev. Lett. (1999), in press; LANL preprint condmat/9905288.

[12] Ll. Serra, A. Puente and E. Lipparini, Phys. Rev. B: Rapid Comm. (1999), in press; LANL preprint cond-mat/9909135.

[13] I. I. Magnúsdóttir and V. Gudmundsson, LANL preprint cond-mat/9907216.

[14] D. A. Broido, K. Kempa, and P. Bakshi, Phys. Rev. B 42.

[15] V. Gudmundsson and R. R. Gerhardts, Phys. Rev. B 43, 12098 (1991); V. Gudmundsson and J. J. Palacios, Phys. Rev. B 52, 11266 (1995).

[16] M. Ferconi and G. Vignale, Phys. Rev. B 50, 14722 (1994).

[17] M. Pi, M. Barranco, A. Emperador, E. Lipparini and Ll. Serra, Phys. Rev. B 57, 14783.

[18] E. Lipparini and Ll. Serra, Phys. Rev. B 57, 6830 (1998); Ll. Serra, M. Barranco, A. Emperador, M. Pi and E. Lipparini, Phys. Rev. B 59, 15290 (1999).

[19] P. A. Maksym and T. Chakraborty, Phys. Rev. Lett. 65, 108 (1990).

[20] S.-R. E. Yang, A. H. MacDonald and M. D. Johnson, Phys. Rev. Lett. 71, 3194 (1993).

[21] P. L. McEuen, E. B. Foxman, J. Kinaret, U. Meirav, M. A. Kastner, N. S. Windgreen, S. J. Wind, Phys. Rev. B 45,11419 (1992).

[22] I. K. Marmokos and C. W. Beenaker, Phys. Rev. B 46, 15562 (1992).

[23] E. H. Lieb, J. P. Solovej and J. Ingvason, Phys. Rev. B 51, 10646 (1995).

[24] N. Barberan, Phys. Rev. B 58, 12970 (1998).

[25] M. Brack and R. K. Bhaduri, Semiclassical Physics (Addison-Wesley, USA, 1997).

[26] B. Tanatar and D. M. Ceperley, Phys. Rev. B 39, 5005 (1989).

[27] In modified atomic units one imposes: $\hbar=1, m=1$ and $e^{2} / \varepsilon=1$. Using $m$ and $\varepsilon$ values corresponding to GaAs the energy, length and time units are $\mathrm{H}^{*} \approx 12 \mathrm{meV}, a_{0}^{*} \approx 98 \AA$ and $\tau^{*} \approx 55 \mathrm{fs}$, respectively.

[28] M. Brack, Rev. Mod. Phys. 65 677, (1993); and references therein.

[29] P. Ring and P. Schuck, The Nuclear Many-Body Problem (Springer, New York, 1980).

[30] A. Domps, P.-G. Reinhard, and E. Suraud, Phys. Rev. Lett. 80, 5520 (1998).

[31] Ll. Serra and E. Lipparini, Europhys. Lett. 40, 667 (1997).

[32] J. F. Dobson, Phys. Rev. Lett. 73, 2244 (1994). 


\section{FIGURES}

FIG. 1. Radial electronic densities for circularly symmetric parabolas in the TFW model (dashed) in comparison with the corresponding KS densities (solid). Panel (a) corresponds to a fixed parabola with $\omega_{0}=0.21 \mathrm{H}^{*}$, that with the parameterization used in the text is given by $N_{p}=40$ and $r_{s}=1.51 a_{0}^{*}$, with different number of electrons. Ordered vertically at $r=0$ are shown $N=2,6,12,20,30,42,56,72$ and 90, respectively. Panel (b) corresponds to a fixed number of electrons $N=42$ and varying parabolas with the same $r_{s}$ and $N_{p}=20,30,40,50$ and 60 (also ordered vertically at $r=0$ ). Panel (c) shows the density for a fixed dot, $N=40$ and $N_{p}=42$ within KS and for varying Weizsäcker coefficient $\lambda=0.25,0.75$ and 1.5 (in vertical order around $r=14)$.

FIG. 2. Energy per electron, as a function of electron number $N$, in the TFW model (smooth line) and in the KS model (symbols). Panels (a) and (b) contain the results for the corresponding panels in Fig. 1.

FIG. 3. Results for neutral jellium disks with $r_{s}=1.51 a_{0}^{*}$. Panel (a) shows the radial densities within the TFW (dashed) and KS (solid). With increasing edge radius the results correspond to $N=12,20,46,58$ and 72. Panel (b) shows the corresponding energies for KS (symbols) and TFW (smooth line).

FIG. 4. Comparison of KS and TFW densities for anisotropic parabolas with $N=20, N_{p}=20$ and $r_{s}=1.51 a_{0}^{*}$ for different deformation parameters. The elliptic contour lines correspond to the semiclassical densities while the coloured background regions show the KS results. The scale bar indicates the density associated with each colour as well as its value for the semiclassical contour lines, which correspond approximately to the colour transitions.

FIG. 5. Dipole excitation spectra, in logarithmic arbitrary units, for charge density and spin density excitations in a parabolic quantum dot. In each panel, the upper curve corresponds to the microscopic RPA calculation and the lower one to the present semiclassical approximation. In the upper panel the triangle shows the value of the parabola coefficient $\omega_{0}$.

FIG. 6. Same as Fig. 4 for a jellium disk with $r_{s}=1.51 a_{0}^{*}$. In this case the triangle corresponds to the parabola that fits the disk potential at the origin.

FIG. 7. Dipole spectra for the parabolic quantum dots of Fig. 4. Solid and dashed lines show the results for oscillations in $x$ and $y$ directions, respectively. For the CDE (two upper panels) we plot the TFW result and the position of the $\omega_{x}$ and $\omega_{y}$ parameters while for the SDE (four lower panels) we compare with the corresponding KS curves (displaced vertically above the TFW ones). As before, vertical scales are in logarithmic arbitrary units.

FIG. 8. Same as Fig. 7 for a parabolic dot with $N=72$ and $N_{p}=72$. The microscopic KS results are not available for $\beta=0.75$. 


\section{TABLES}

TABLE I. Energies per electron (in $\mathrm{H}^{*}$ ) within the TFW and KS models. The results correspond to a parabola with $N=20, N_{p}=20, r_{s}=1.51 a_{0}^{*}$ and different deformations. See text.

\begin{tabular}{c|ccccc}
\hline \hline & & & & & \\
& 1 & 0.875 & 0.75 & 0.625 & 0.5 \\
\hline KS & -3.026 & -3.032 & -3.059 & -3.118 & -3.223 \\
TFW & -3.025 & -3.033 & -3.061 & -3.119 & -3.225 \\
\hline \hline
\end{tabular}

